Edinburgh $97 / 24$

FTUV/98-44

IFIC $/ 98-45$

ROME1-1184/97

$\mathrm{SNS} / \mathrm{PH} / 1998-010$

\title{
Non-perturbative Renormalization of Quark bilinears
}

\author{
V. Giménez \\ Dep. de Fisica Teorica and IFIC, Univ. de València, \\ Dr. Moliner 50, E-46100, Burjassot, València, Spain \\ L. Giusti \\ Scuola Normale Superiore, P.zza dei Cavalieri 7, I-56100 Pisa, Italy \\ INFN Sezione di Pisa, I-56100 Pisa, Italy \\ F. Rapuano \\ Dipartimento di Fisica, Università di Roma 'La Sapienza' and \\ INFN, Sezione di Roma, P.le A. Moro 2, I-00185 Roma, Italy. \\ M. Talevi \\ Department of Physics \& Astronomy, University of Edinburgh \\ The King's Buildings, Edinburgh EH9 3JZ, UK
}

\begin{abstract}
We compute non-perturbatively the renormalization constants of quark bilinears on the lattice in the quenched approximation at three values of the coupling $\beta=6 / g_{0}^{2}=$ 6.0,6.2, 6.4 using both the Wilson and the tree-level improved SW-Clover fermion action. We perform a Renormalization Group analysis at the next-to-next-to-leading order and compute Renormalization Group invariant values for the constants. The results are applied to obtain a fully non-perturbative estimate of the vector and pseudoscalar decay constants.
\end{abstract}

PACS: 11.15.H, 12.38.Gc, 13.30.Eg, 14.20.-c and 14.40.-n 


\section{Introduction}

Lattice QCD is an unique tool to compute non-perturbatively from first principles the mass spectrum, leptonic decay constants and in general hadronic matrix elements of local operators. Renormalization constants, relating the operators on the lattice to the continuum are necessary to extract physical informations from Monte Carlo simulations. In this paper we study the renormalization properties of composite bilinear operators $[1,2]$ with the quark action discretized a lá Wilson.

In principle, the renormalization of quark bilinears can be computed in oneloop perturbation theory, as there are no power divergences [3,4]. It is well known, though, that lattice perturbation theory is ill behaved, due to the presence of tadpole-like diagrams $[5,6]$ and at values of the coupling $\beta=6 / g_{0}^{2}=$ $6.0-6.4$, the higher-order corrections may not be small, thus introducing a large uncertainty in the calculation of the renormalized matrix elements in some continuum scheme. These problems are avoided using non-perturbative (NP) renormalization techniques $[7,8]$. The procedure proposed in [7] allows a full non-perturbative computation of the matrix elements of composite operators in the Regularization Independent (RI) scheme $[7,9]$. The matching between the RI scheme and $\overline{\mathrm{MS}}$, which is intrinsically perturbative, is computed using only continuum perturbation theory, which is well behaved. This method has been shown to be quite successful in reproducing results obtained by other methods, such as chiral Ward Identities [10]. Quite impressive is also the influence of the NP renormalization in the measurement of the quark masses [11], the chiral condensate [12] and, for four-fermion operators, in the restoration of the correct chiral behaviour of the $B$-parameters in weak decays [13-15]. Moreover, any attempt to tackle the question of the $\Delta I=1 / 2$ rule must rely on NP methods [16].

In this paper, we extend the exploratory computations done in [7] to a high statistics study with both the standard Wilson action and the tree-level improved SW-Clover action [17] at different values of the coupling in the quenched approximation. We compare the dependence on the renormalization scale of the renormalized operators with respect to the solution predicted from the Renormalization Group Equation (RGE) at the next-to-next-to-leading-order (NNLO). Moreover, we use this analysis to estimate the systematics due to discretization errors on the renormalization constants.

Recently, there has been much progress in the Symanzik on-shell improvement program [18], obtaining a non-perturbative determination of the renormalization constants with a fully $O(a)$ improved action $[19,20]$. An extension of this program, to take into account all terms of $O(a)$ including the ones proportional to the quark mass, has been proposed in [21]. Whereas the non-perturbative 
improvement is the direction of future, it is true that Wilson and tree-level Clover actions are still widely used, e.g. for four-fermion operators relevant to weak decays (cf. for example ref. [22] and references therein). Moreover, the study of the renormalization properties in the chiral limit with an unimproved and partially improved action will provide further insight for the discretization effects in lattice QCD.

The outline of this paper is as follows. In sec. 2 we review the non-perturbative method (NPM) proposed in ref. [7] and set the notation for the remainder of this work. The Renormalization Group ( $R G$ ) analysis of the quark bilinears is outlined in sec. 3 , while in sec. 4 we present the numerical results for the renormalization constants and discuss the systematic errors. In sec. 5 we apply these results to the the computation of the leptonic decay constants of the vector and pseudoscalar mesons. We finish with our conclusions in sec. 6 .

\section{Non-perturbative renormalization method}

In this section we review the method of ref. [7], which we have used to compute non-perturbatively the renormalization constants of quark bilinears in the Regulatization Independent (RI) scheme [9]. The method imposes renormalization conditions non-perturbatively, directly on quark and gluon Green functions, in a fixed gauge, with given off-shell external states of large virtuality. Notice that in RI the renormalization conditions are independent of the regularization scheme but they depend on the external states and on the gauge used in the procedure.

The renormalization scale $\mu^{2}$, determined from the virtuality of the external states $p^{2}$, must satisfy the condition $\Lambda_{\mathrm{QCD}} \ll \mu \ll O(1 / a)$, see [7].

We have worked in the lattice Landau gauge, defined by minimizing the functional

$$
\operatorname{Tr}\left[\sum_{\mu=1}^{4}\left(U_{\mu}(x)+U_{\mu}^{\dagger}(x)\right)\right]
$$

The necessity to fix the gauge introduces a systematic uncertainty due to the existence of both continuum and lattice Gribov copies [24] and the numerical noise that they can generate. These effects are expected to die off at large virtuality and on the renormalization of two-quark operators have been found to be small, comparable to the statistical noise [23]. We are making the assumption that the Landau lattice gauge-fixing procedure brings gauge fixed lattice operators into the corresponding continuum ones as $a \rightarrow 0$ [25]. 
Let us consider a local lattice quark bilinear $O_{\Gamma}=\bar{q} \Gamma q$, where $\Gamma$ is a Dirac matrix $\rrbracket$. The renormalization condition is imposed on the amputated Green function computed between off-shell quark states of momentum $p$ in the Landau gauge

$$
\Lambda_{\Gamma}(p a)=S_{q}(p a)^{-1} G_{\Gamma}(p a) S_{q}(p a)^{-1}
$$

where $G_{\Gamma}(p a)$ and $S_{q}(p a)$ are the non-amputated Green function and quark propagator, calculated non-perturbatively via Monte Carlo simulations [7]. The renormalization constant $Z_{\Gamma}^{\mathrm{RI}}\left(\mu a, g_{0}\right)$ of $O_{\Gamma}$, in the RI scheme, is determined by the condition

$$
\left.Z_{\Gamma}^{\mathrm{RI}}(\mu a) Z_{q}^{-1}(\mu a) \operatorname{Tr} \mathrm{P}_{\Gamma} \Lambda_{\Gamma}(p a)\right|_{p^{2}=\mu^{2}}=1
$$

and the renormalized operator is related to the bare one by $\hat{O}_{\Gamma}^{\mathrm{RI}}=Z_{\Gamma}^{\mathrm{RI}} O_{\Gamma}$. In eq. (3) $\mathrm{P}_{\Gamma}$ is a suitable projector on the tree-level amputated Green function. In the case of the quark bilinears the projector is simply proportional to $\Gamma^{\dagger}$. $Z_{q}$ is the wave function renormalization which can be defined from the Ward Identity (WI) as [7]

$$
Z_{q}(\mu a)=-\left.i \frac{1}{12} \operatorname{Tr}\left[\frac{\partial S(p a)^{-1}}{\partial p^{\prime}}\right]\right|_{p^{2}=\mu^{2}}
$$

To avoid derivatives with respect to a discrete variable, we have used

$$
Z_{q}^{\prime}(\mu a)=-\left.i \frac{1}{12} \frac{\operatorname{Tr} \sum_{\mu=1,4} \gamma_{\mu} \sin \left(p_{\mu} a\right) S(p a)^{-1}}{4 \sum_{\mu=1,4} \sin ^{2}\left(p_{\mu} a\right)}\right|_{p^{2}=\mu^{2}}
$$

which, in the Landau Gauge, differs from $Z_{q}$ by a finite term of order $\alpha_{s}^{2}$. The matching coefficient can be computed using continuum perturbation theory only, and up to order $\alpha_{s}^{2}[26]$

$$
\frac{Z_{q}}{Z_{q}^{\prime}}=1-\frac{\alpha_{s}^{2}}{(4 \pi)^{2}} \Delta_{q}^{(2)}+\ldots
$$

with, in the Landau gauge,

$$
\Delta_{q}^{(2)}=\frac{\left(N_{c}^{2}-1\right)}{16 N_{c}^{2}}\left(3+22 N_{c}^{2}-4 N_{c} n_{f}\right) .
$$

$\overline{1}$ In the following, we shall denote with $\Gamma=A, V, P, S$ the axial and vector currents and the pseudoscalar and scalar densities. 
where $N_{c}$ is the number of colours and $n_{f}$ the number of active quarks. Eqs. (3) and (5) define the constants $Z_{\Gamma}^{\prime} \mathrm{RI}$. From eq. (6) we obtain for $Z_{\Gamma}^{\mathrm{RI}}$

$$
Z_{\Gamma}^{\mathrm{RI}}(\mu a)=\left(1-\frac{\alpha_{s}^{2}(\mu)}{(4 \pi)^{2}} \Delta_{q}^{(2)}\right) Z_{\Gamma}^{\mathrm{RI}}(\mu a),
$$

which satisfy the Ward identities at the NNLO. The matching between RI and $\overline{\mathrm{MS}}, N D R$ requires continuum perturbation theory only $[7,9]$. Since both RI and $\overline{\mathrm{MS}}, N D R$ respect chirality and the renormalized operators with the correct chiral behaviour are unique, we have $Z_{A}^{\mathrm{RI}}=Z_{A}^{\overline{\mathrm{MS}}}$ and $Z_{V}^{\mathrm{RI}}=Z_{V}^{\overline{\mathrm{MS}}}$. For the same reason

$$
\frac{Z_{S}^{\mathrm{RI}}}{Z_{S}^{\overline{\mathrm{MS}}}}=\frac{Z_{P}^{\mathrm{RI}}}{Z_{P}^{\overline{\mathrm{MS}}}}=\frac{Z_{m}^{\overline{\mathrm{MS}}}}{Z_{m}^{\mathrm{RI}}}
$$

where $Z_{m}$ is the quark mass renormalization. In ref. [26] $Z_{m}^{\overline{\mathrm{MS}}} / Z_{m}^{\mathrm{RI}}$ has been computed up to order $\alpha_{s}^{2}$. Using eq. (9) one can compute at the same order the matching coefficients of the scalar and pseudoscalar densities

$$
Z_{\Gamma}^{\overline{\mathrm{MS}}}(\mu)=\left(1+\frac{\alpha_{s}(\mu)}{4 \pi} C^{(1)}+\frac{\alpha_{s}^{2}(\mu)}{(4 \pi)^{2}} C^{(2)}\right) Z_{\Gamma}^{\mathrm{RI}}(\mu)
$$

where $\Gamma=P, S$ and in the Landau gauge,

$$
\begin{aligned}
C^{(1)}= & \frac{8\left(N_{c}^{2}-1\right)}{4 N_{c}}, \\
C^{(2)}=\frac{\left(N_{c}^{2}-1\right)}{96 N_{c}^{2}}( & -309+3029 N_{c}^{2} \\
& \left.-288 \zeta_{3}-576 N_{c}^{2} \zeta_{3}-356 N_{c} n_{f}\right) .
\end{aligned}
$$

where $\zeta_{3}=1.20206 \ldots$. The dependence on the gauge and the external states of the RI scheme will cancel with the corresponding dependence of the matching coefficients in eq. (10), up to higher orders in continuum perturbative expansion and up to discretization errors.

\section{Renormalization Group Analysis}

The RGE expresses a general property of the Green's functions of a renormalized theory and therefore they are valid non-perturbatively. To study the RG properties of bilinears we work in the $\overline{\mathrm{MS}}$ scheme and in the Landau gauge. 
The generic, forward, renormalized two-point Green's function, computed between quark states of virtuality $p^{2}$ obeys the RGE

$$
\left[\mu^{2} \frac{d}{d \mu^{2}}+\frac{\gamma_{\Gamma}}{2}\right] \Gamma\left(\frac{p}{\mu}\right)=\left[\mu^{2} \frac{\partial}{\partial \mu^{2}}+\beta\left(\alpha_{s}\right) \frac{\partial}{\partial \alpha_{s}}+\frac{\gamma_{\Gamma}}{2}\right] \Gamma\left(\frac{p}{\mu}\right)=0,
$$

where the QCD $\beta$-function and the anomalous dimension of the renormalized operator $\hat{O}_{\Gamma}$ are gauge invariant to all orders in perturbation theory and are defined as:

$$
\begin{gathered}
\frac{\beta\left(\alpha_{s}\right)}{4 \pi}=\mu^{2} \frac{d}{d \mu^{2}}\left(\frac{\alpha_{s}}{4 \pi}\right)=-\sum_{i=0}^{\infty} \beta_{i}\left(\frac{\alpha_{s}}{4 \pi}\right)^{i+2} \\
\gamma_{\Gamma}\left(\alpha_{s}\right)=-2 Z_{\Gamma}^{-1} \mu^{2} \frac{d}{d \mu^{2}} Z_{\Gamma}=\sum_{i=0}^{\infty} \gamma_{\Gamma}^{(i)}\left(\frac{\alpha_{s}}{4 \pi}\right)^{i+1} .
\end{gathered}
$$

In a continuum regularization which respects chirality the axial and vector currents do not get renormalized, i.e. $Z_{A}=Z_{V}=1$, as can be easily shown through the Ward Identities that they satisfy. Since $m(\mu) P(\mu)$, with $P(\mu)$ the pseudoscalar density, is renormalization group invariant, the scalar and pseudoscalar densities have renormalization constants which obey $Z_{S}=Z_{P}=$ $1 / Z_{m} \longleftarrow$. Therefore one can express the anomalous dimension $\gamma_{\Gamma}$ of bilinear operators as a function of $\gamma_{m}$ :

$$
\begin{aligned}
& \gamma_{A}=\gamma_{V}=0 \\
& \gamma_{P}=\gamma_{S}=-\gamma_{m}
\end{aligned}
$$

To solve the RGE's in the NNLO approximation, the expansions of the $\beta$ function and anomalous dimension up to three loops is required.

The running of the coupling constant $\alpha_{s}^{\overline{\mathrm{MS}}}$ is given by

$$
\begin{aligned}
\frac{\alpha_{s}^{\overline{\mathrm{MS}}}}{4 \pi}\left(q^{2}\right)= & \frac{1}{\beta_{0} \ln \left(q^{2}\right)}-\frac{\beta_{1}}{\beta_{0}^{3}} \frac{\ln \ln \left(q^{2}\right)}{\ln ^{2}\left(q^{2}\right)} \\
& +\frac{1}{\beta_{0}^{5} \ln ^{3}\left(q^{2}\right)}\left(\beta_{1}^{2} \ln ^{2} \ln \left(q^{2}\right)-\beta_{1}^{2} \ln \ln \left(q^{2}\right)+\beta_{2}^{\overline{\mathrm{MS}}} \beta_{0}-\beta_{1}^{2}\right)
\end{aligned}
$$

where $q^{2}=\left(\mu / \Lambda_{\mathrm{QCD}}^{\overline{\mathrm{MS}}}\right)^{2}$. For the continuum $\overline{\mathrm{MS}}$ scale parameter $\Lambda_{\mathrm{QCD}}^{\overline{\mathrm{MS}}}$ at the NNLO, in the quenched approximation, we have used $\Lambda_{\mathrm{QCD}}^{\overline{\mathrm{MS}}}=0.251 \pm$ $0.021 \mathrm{GeV}[20]$. The QCD $\beta$-function is scheme independent only up to two

$\overline{2}$ We stress that we are studying the RGE evolution in a continuum renormalization scheme. 
loops. The additional term of the expansion has been computed in the $\overline{\mathrm{MS}}$ scheme in [27]:

$$
\begin{aligned}
\beta_{0}= & \frac{11}{3} N_{c}-\frac{2}{3} n_{f}, \\
\beta_{1}= & \frac{34}{3} N_{c}^{2}-\frac{10}{3} N_{c} n_{f}-\frac{\left(N_{c}^{2}-1\right)}{N_{c}} n_{f}, \\
\beta_{2}^{\overline{\mathrm{MS}}}= & \frac{2857}{54} N_{c}^{3}+\frac{\left(N_{c}^{2}-1\right)^{2}}{4 N_{c}^{2}} n_{f}-\frac{205}{36}\left(N_{c}^{2}-1\right) n_{f} \\
& -\frac{1415}{54} N_{c}^{2} n_{f}+\frac{11}{18} \frac{\left(N_{c}^{2}-1\right)}{N_{c}} n_{f}^{2}+\frac{79}{54} N_{c} n_{f}^{2}
\end{aligned}
$$

The mass anomalous dimension in the $\overline{\mathrm{MS}}$ scheme up to three loops is given by $[28,29]$ :

$$
\begin{aligned}
\gamma_{m}^{(0)}= & 3 \frac{N_{c}^{2}-1}{N_{c}} \\
\gamma_{m}^{(1)}= & \frac{N_{c}^{2}-1}{N_{c}^{2}}\left(-\frac{3}{4}+\frac{203}{12} N_{c}^{2}-\frac{5}{3} N_{c} n_{f}\right), \\
\gamma_{m}^{(2)}= & \frac{N_{c}^{2}-1}{N_{c}^{3}}\left[\frac{129}{8}-\frac{129}{8} N_{c}^{2}+\frac{11413}{108} N_{c}^{4}\right. \\
& \left.+n_{f}\left(\frac{23}{2} N_{c}-\frac{1177}{54} N_{c}^{3}-12 N_{c} \zeta_{3}-12 N_{c}^{3} \zeta_{3}\right)-\frac{35}{27} N_{c}^{2} n_{f}^{2}\right],
\end{aligned}
$$

where $\zeta$ is the Riemann zeta function.

The evolution of the renormalized bilinear operators is determined by eqs. (12) and (16). The solution can be expressed in the $\overline{\mathrm{MS}}$ scheme in the form [30]

$$
\hat{O}_{\Gamma}^{\overline{\mathrm{MS}}}(\mu)=\frac{c_{\Gamma}^{\overline{\mathrm{MS}}}(\mu)}{c_{\Gamma}^{\overline{\mathrm{MS}}}\left(\mu_{0}\right)} \hat{O}_{\Gamma}^{\overline{\mathrm{MS}}}\left(\mu_{0}\right),
$$

where

$$
\begin{aligned}
c_{\Gamma}^{\overline{\mathrm{MS}}}(\mu) & =\alpha_{s}(\mu)^{\bar{\gamma}_{\Gamma}^{(0)}}\left\{1+\frac{\alpha_{s}}{4 \pi}\left(\bar{\gamma}_{\Gamma}^{(1)}-\bar{\beta}_{1} \bar{\gamma}_{\Gamma}^{(0)}\right)\right. \\
& \left.+\frac{1}{2}\left(\frac{\alpha_{s}(\mu)}{4 \pi}\right)^{2}\left[\left(\bar{\gamma}_{\Gamma}^{(1)}-\bar{\beta}_{1} \bar{\gamma}_{\Gamma}^{(0)}\right)^{2}+\bar{\gamma}_{\Gamma}^{(2)}+\bar{\beta}_{1}^{2} \bar{\gamma}_{\Gamma}^{(0)}-\bar{\beta}_{1} \bar{\gamma}_{\Gamma}^{(1)}-\bar{\beta}_{2} \bar{\gamma}_{\Gamma}^{(0)}\right]\right\},
\end{aligned}
$$

with $\bar{\beta}_{i}=\beta_{i} / \beta_{0}$ and $\bar{\gamma}_{\Gamma}^{(i)}=\gamma_{\Gamma}^{(i)} /\left(2 \beta_{0}\right)$.

By using the eqs. (9) and (19), the evolution of the bilinear quark operators 
at the NNLO in the RI scheme becomes

$$
\hat{O}_{\Gamma}^{\mathrm{RI}}(\mu)=\frac{c_{\Gamma}^{\mathrm{RI}}(\mu)}{c_{\Gamma}^{\mathrm{RI}}\left(\mu_{0}\right)} \hat{O}_{\Gamma}^{\mathrm{RI}}\left(\mu_{0}\right),
$$

where

$$
c_{\Gamma}^{\mathrm{RI}}(\mu)=\frac{Z_{\Gamma}^{\mathrm{RI}}(\mu)}{Z_{\Gamma}^{\overline{\mathrm{MS}}}(\mu)} c_{\Gamma}^{\overline{\mathrm{MS}}}(\mu) .
$$

Eqs. (21) and (22) define the evolution at NNLO in the RI scheme of the renormalization constants with the scale $\mu$. In order to compare with the numerical NP results we define a Renormalization Group Invariant (RGI) constant as

$$
Z_{\Gamma}^{\mathrm{RGI}}(a)=\frac{Z_{\Gamma}^{\prime \mathrm{RI}}\left(\mu a, m_{q} a=0\right)}{c_{\Gamma}^{\prime \mathrm{RI}}(\mu)},
$$

where

$$
c_{\Gamma}^{\prime \mathrm{RI}}(\mu)=\frac{Z_{\Gamma}^{\prime \mathrm{RI}}(\mu)}{Z_{\Gamma}^{\mathrm{RI}}(\mu)} c_{\Gamma}^{\mathrm{RI}}(\mu)
$$

takes into account the mismatch between $Z_{q}$ and $Z_{q}^{\prime}$, cf. eq (6). Up to higher order terms in continuum perturbation theory and up to discretization errors, $Z_{\Gamma}^{\mathrm{RGI}}(a)$ should be independent of $\mu$, in the region in which perturbation theory is valid, i.e. $\mu \gtrsim 2 \mathrm{GeV}$, independent of the renormalization scheme, of the external states and gauge invariant. Being the continuum evolution already at NNLO, we assume any scale dependence to be dominated by discretization effects. As an estimate of this systematic error we will take the semidispersion of the values of the renormalization constants in the perturbative region.

\section{Non-perturbative renormalization constants}

The renormalization constants for the bilinears presented in this paper are obtained at three different gauge couplings $g_{0}^{2}$, corresponding to $\beta=6 / g_{0}^{2}=$ 6.0,6.2 and 6.4 using both the standard Wilson action and the tree-level improved SW-Clover fermion action [17] 3 . A summary of the parameters used

3 We note that in our implementation of the tree-level improvement program the relationship $Z_{S}=1 / Z_{m}$ is not true anymore because we have used non-local " $D$ rotated" operators; we refer to $[7,11]$ for details. 


\begin{tabular}{c|cccccc|}
\hline \hline$\beta$ & 6.0 & 6.0 & 6.2 & 6.2 & 6.4 & 6.4 \\
Action & SW & Wilson & SW & Wilson & SW & Wilson \\
\# Confs & 100 & 100 & 180 & 100 & 60 & 60 \\
Volume & $16^{3} \times 32$ & $16^{3} \times 32$ & $16^{3} \times 32$ & $16^{3} \times 32$ & $24^{3} \times 32$ & $24^{3} \times 32$ \\
\hline$\kappa$ & 0.1425 & 0.1530 & 0.14144 & 0.1510 & 0.1400 & 0.1488 \\
& 0.1432 & 0.1540 & 0.14184 & 0.1515 & 0.1403 & 0.1492 \\
& 0.1440 & 0.1550 & 0.14224 & 0.1520 & 0.1406 & 0.1496 \\
& & & 0.14264 & 0.1526 & 0.1409 & 0.1500 \\
\hline$\kappa_{\text {crit }}$ & 0.14551 & 0.15683 & 0.14319 & 0.15337 & 0.14143 & 0.15058 \\
\hline \hline
\end{tabular}

Table 1

Summary of parameters used in the non-perturbative calculation of the renormalization constants.

in the NP calculation of the renormalization constants is presented in tab. 1. The errors have been obtained with the jacknife method, decimating 10 configurations at a time. The lattice scale $a^{-1}$ for the different couplings has been determined from $M_{K^{*}}[34]$ and is shown in tab. 5 .

\subsection{Axial and vector currents}

Let us consider first the axial and vector currents. Since each obeys a chiral Ward Identity [1] their renormalization constants are finite, i.e. scale independent. In figs. 1 and 2 we show $Z_{A}$ and $Z_{V}$, calculated in the RI scheme in the chiral limit for both the SW and Wilson actions, as a function of the renormalization scale $\mu$. As explained in [7], we expect to find a "window" in the range of values of $\mu$ in which the $Z$ 's are scale independent. Since at large values of $\mu$ the $Z$ 's will be more sensible to discretization errors, we expect the window to be wider as we approach the continuum.

We can clearly see that these expectations are satisfied by the data shown in figs. 1 and 2, and also reported in tabs. 2 and 3. For the axial and vector currents, the RGI values given in the tables are simply the values of the constants calculated at $\mu a \simeq 1$, since $c_{\Gamma}^{\prime R I}(\mu)$ for $Z_{A}$ and $Z_{V}$ is totally negligible in comparison with the final error. The first error is statistical, while the second is the systematic error estimated as the semidispersion of the values in the region in which we believe perturbation theory to be reliable, i.e. $\mu \gtrsim 2 \mathrm{GeV}$. In the SW case, the axial current shows a more pronounced systematic effect compared to the vector current, reflected in a reduced stability in $\mu$ of the plots. In the Wilson case, on the other hand, it's the vector current that is more fluctuating in the scale $\mu$. Moreover, as far the comparison between the 

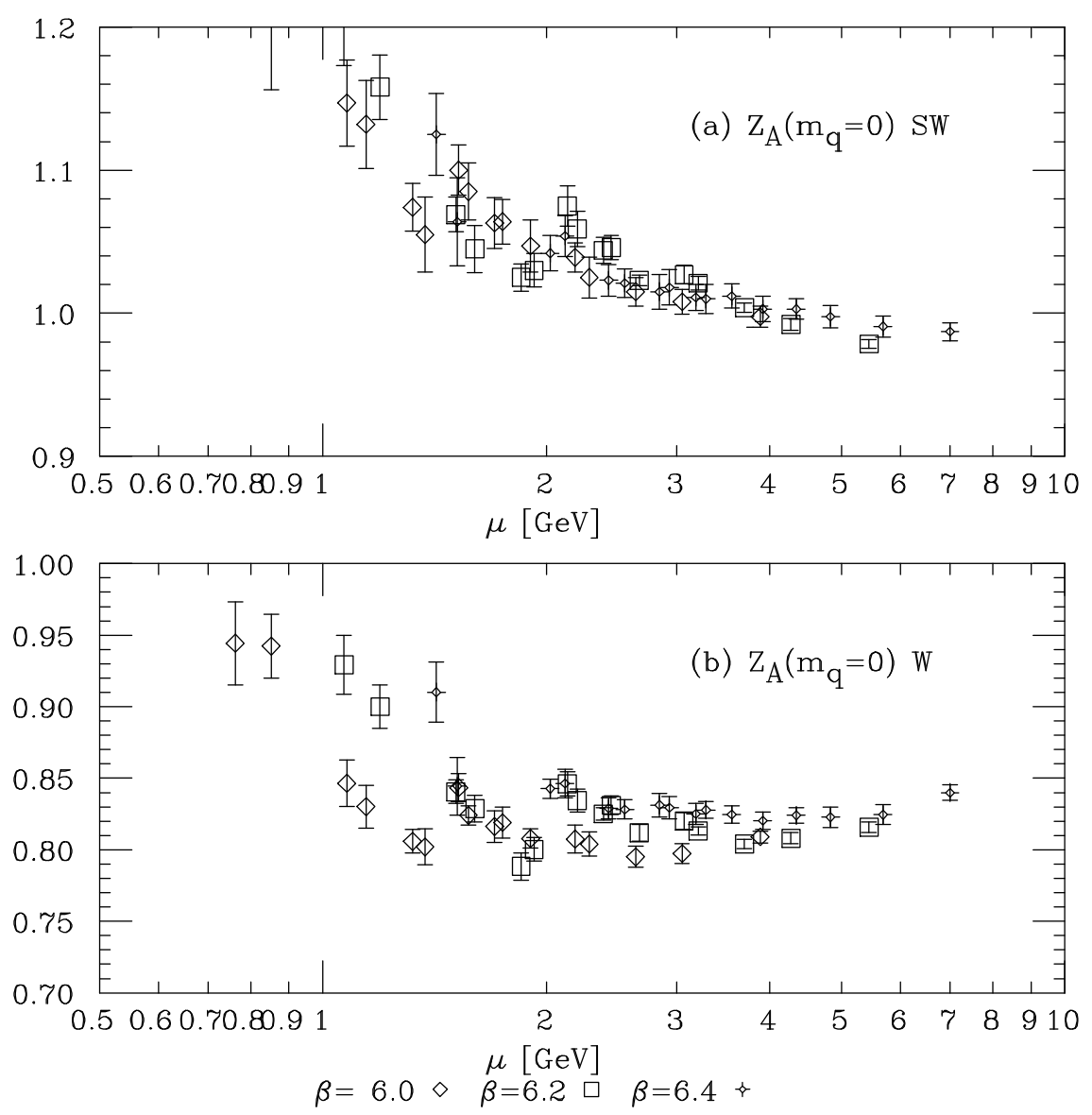

Fig. 1. Renormalization of the axial current for (a) SW and (b) Wilson action as a function of $\mu$ for all couplings. The lattice spacing is determined from $M_{K^{*}}$.

SW and Wilson actions is concerned (at the same value of $\beta$ ), we note that for the axial current the Wilson action seems more stable than the SW one, while for the vector current it's the SW action which shows a more pronounced plateau.

In tab. 4 we also give the values of $Z_{A}$ and $Z_{V}$ determined from the WI's $[2,31,32]$. Caution should be used in comparing the values obtained from the NPM and the WI's as the latter are sometimes available only for finite values of the quark mass, but since the mass dependence is expected to be weak, it is still a significant check. The agreement between the NPM and the WI's is good for the axial current in the SW case, around $\mu a \approx 1$, as already found in [7]. On the other hand, in the Wilson case the WI value at $\beta=6.0$ is even larger than the NPM at $\beta=6.4$, although the error quoted from the WI is so large $(\approx 10 \%)$ that a significant comparison is not possible. For the vector current, we notice that in SW case the NP values are slightly higher than the WI although the discrepancy tends to diminish as $\beta$ increases, whereas in the Wilson case, the comparison is more complicated. This is due to the well-know discretization errors which affect the hadronic matrix elements of the vector 

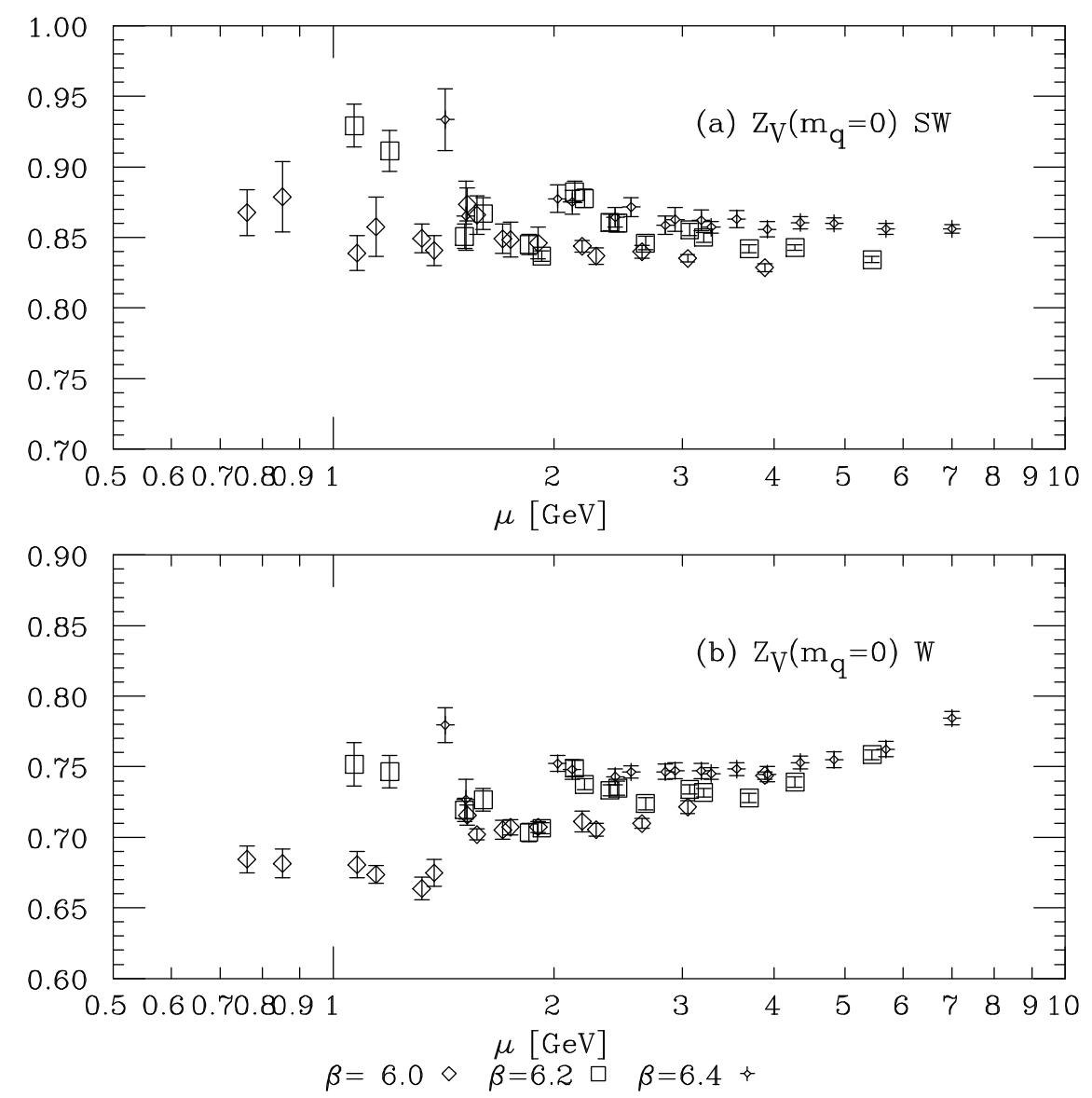

Fig. 2. Renormalization of the vector current for (a) SW and (b) Wilson action as a function of $\mu$ for all couplings. The lattice spacing is determined from $M_{K^{*}}$.

current, in particular of the conserved one [31]. We expect the values of $Z_{A}$ and $Z_{V}$ to approach unity as move towards the continuum limit and the coupling goes to zero. All the data obtained with the NPM support this conclusion.

In tab. 4 we also present the one-loop perturbative values [3,4], evaluated at $\mu a=1$ in the RI scheme according to eq. (10). We present both Stardard PT (SPT), in which the bare coupling $g_{0}^{2}$ is used as the expansion parameter, and Boosted PT (BPT) in which the coupling used is [6]

$$
\alpha_{s}^{\mathrm{BPT}}=\frac{1}{\langle\square\rangle} \alpha_{s}^{\text {latt }}=\frac{1}{\langle\square\rangle} \frac{g_{0}^{2}}{4 \pi}
$$

where $\langle\square\rangle$ is the expectation value of the plaquette. In all the perturbative calculations, the effects coming from the discretization of loop integrals on a finite lattice have been ignored. As already emphasized in [7], perturbation theory does not agree with the non-perturbative values, except in a number of limited cases, as the vector current. In the case of the pseudoscalar density, the failure is quite dramatic and has important phenomenological consequences on 
the determination of the quark masses and the quark condensate, as explained in refs. [11]. Moreover, compared to the NPM, the perturbative values show a less pronounced variation with the coupling. Thus, even if the agreement improves as we approach the continuum, the discrepancy remains sizable even at the smallest coupling.

The exploratory numerical calculations in [7] were carried out at $\beta=6.0$ and a single value of the hopping parameter $\kappa=0.1425$, corresponding to $m_{q} a \simeq 0.07$. We have checked that the results are compatible with ours within statistical errors. We have chosen not to separate the time and space components of the currents as they agree within errors. It is worth noting that the sizable difference between the renormalization constants of the different components and the large fluctuations found in [7] at large values of $\mu^{2} a^{2}$ were not due to strong discretization effects, but to the choice of the external momenta. In fact, the components of the momenta $p=\left(p_{0}, p_{1}, p_{2}, p_{3}\right)$ were chosen with large differences among the $p_{i}$ 's, thus greatly enhancing the rotational symmetry breaking on the hypercubic lattice.

Recently, the non-perturbative method of ref. [7] has also been applied to the renormalization of bilinears in ref. [33], in which "momentum" sources and sinks and translational invariance are used to reduce the statistical noise in the determination of the $Z$ 's. The method has been tested for Wilson fermions at $\beta=6.0$, and the values of the $Z$ 's in the chiral limit calculated at $\mu a \simeq 1$ are $Z_{A}=0.7807(8), Z_{V}=0.6822(7), Z_{P}=0.4357(17)$ and $Z_{S}=0.6808(15)$. The errors quoted are purely statistical and are much smaller than the systematic errors coming from the discretization effects, which are expected to be quite large in the Wilson case. With this in mind, we find that the agreement between the values of [33] and ours is resonable.

\subsection{Pseudoscalar and scalar densities}

The pseudoscalar and scalar densities differ from the axial and vector currents in that their renormalization is not finite but scale dependent. We show in figs. 3 and 4 the RGI values, computed according to eq. (23). We stress that the RGI values are only reliable from values of $\mu \gtrsim 2 \mathrm{GeV}$, i.e. when continuum perturbation theory is to be trusted. The RGI value in tabs. 2 and 3 has been calculated from $\mu a \simeq 1$. The systematic error is calculated in the same fashion as for the axial and vector currents. It is easily noticeable the difference in the behaviour of $Z_{P}$ and $Z_{S}$ as a function of $\mu$. The former approaches a plateau only at relatively large values of $\mu$, while the latter presents a very clear and long plateau. This behaviour, also reflected in a much larger systematic error for the pseudoscalar, does not depend on the choice of the action. 


\begin{tabular}{|c|c|c|c|c|c|c|}
\hline$\beta$ & $\mu^{2} a^{2}$ & $Z_{A}$ & $Z_{V}$ & $Z_{P}$ & $Z_{S}$ & $Z_{P} / Z_{S}$ \\
\hline \multirow{9}{*}{6.0} & 0.308 & $1.147(30)$ & $0.839(12)$ & $0.199(8)$ & $0.711(36)$ & $0.293(20)$ \\
\hline & 0.617 & $1.100(18)$ & $0.874(12)$ & $0.335(11)$ & $0.799(24)$ & $0.422(16)$ \\
\hline & 0.964 & $1.047(18)$ & $0.846(11)$ & $0.409(8)$ & $0.834(18)$ & $0.492(13)$ \\
\hline & 1.272 & $1.039(10)$ & $0.844(4)$ & $0.457(6)$ & $0.862(18)$ & $0.531(12)$ \\
\hline & 1.388 & $1.025(14)$ & $0.837(6)$ & $0.467(6)$ & $0.871(18)$ & $0.537(14)$ \\
\hline & 1.851 & $1.015(10)$ & $0.840(5)$ & $0.516(6)$ & $0.905(11)$ & $0.570(9)$ \\
\hline & 2.467 & $1.008(9)$ & $0.835(3)$ & $0.555(4)$ & $0.943(12)$ & $0.588(10)$ \\
\hline & 4.010 & $0.998(7)$ & $0.829(3)$ & $0.610(3)$ & $0.993(11)$ & $0.614(8)$ \\
\hline & RGI & $1.047(18)(25)$ & $0.846(11)(9)$ & $0.278(5)(40)$ & $0.567(12)(8)$ & $0.492(13)(61)$ \\
\hline \multirow{9}{*}{6.2} & 0.308 & $1.069(12)$ & $0.851(9)$ & $0.248(8)$ & $0.713(28)$ & $0.372(20)$ \\
\hline & 0.617 & $1.075(14)$ & $0.882(8)$ & $0.401(9)$ & $0.837(21)$ & $0.496(17)$ \\
\hline & 0.964 & $1.023(4)$ & $0.846(5)$ & $0.466(4)$ & $0.851(11)$ & $0.563(8)$ \\
\hline & 1.272 & $1.027(7)$ & $0.856(4)$ & $0.523(4)$ & $0.904(7)$ & $0.581(6)$ \\
\hline & 1.388 & $1.021(5)$ & $0.850(4)$ & $0.527(4)$ & $0.906(10)$ & $0.590(7)$ \\
\hline & 1.851 & $1.004(3)$ & $0.842(3)$ & $0.564(3)$ & $0.928(3)$ & $0.608(4)$ \\
\hline & 2.467 & $0.992(4)$ & $0.843(2)$ & $0.602(3)$ & $0.950(6)$ & $0.630(6)$ \\
\hline & 4.010 & $0.978(3)$ & $0.834(2)$ & $0.642(2)$ & $0.983(4)$ & $0.654(5)$ \\
\hline & RGI & $1.023(4)(24)$ & $0.846(5)(11)$ & $0.295(2)(32)$ & $0.540(7)(9)$ & $0.563(8)(45)$ \\
\hline \multirow{9}{*}{6.4} & 0.313 & $1.042(12)$ & $0.877(10)$ & $0.405(11)$ & $0.739(15)$ & $0.549(18)$ \\
\hline & 0.617 & $1.015(12)$ & $0.859(6)$ & $0.498(8)$ & $0.810(17)$ & $0.616(16)$ \\
\hline & 0.964 & $1.012(9)$ & $0.863(6)$ & $0.555(6)$ & $0.852(13)$ & $0.652(10)$ \\
\hline & 1.169 & $1.003(9)$ & $0.856(5)$ & $0.572(5)$ & $0.869(15)$ & $0.659(11)$ \\
\hline & 1.439 & $1.003(7)$ & $0.861(4)$ & $0.597(6)$ & $0.896(15)$ & $0.667(12)$ \\
\hline & 1.782 & $0.997(8)$ & $0.860(4)$ & $0.618(5)$ & $0.918(13)$ & $0.673(11)$ \\
\hline & 2.467 & $0.991(7)$ & $0.856(4)$ & $0.646(4)$ & $0.946(13)$ & $0.683(9)$ \\
\hline & 3.740 & $0.987(6)$ & $0.856(3)$ & $0.676(4)$ & $0.974(10)$ & $0.694(9)$ \\
\hline & RGI & $1.012(9)(12)$ & $0.863(6)(4)$ & $0.327(4)(17)$ & $0.502(8)(9)$ & $0.652(10)(21)$ \\
\hline
\end{tabular}

Table 2

Non-perturbative values of $Z_{\Gamma}^{\mathrm{RI}}\left(m_{q}=0\right)$ with the SW action, for all couplings at several renormalization scales $\mu^{2} a^{2}$. For the values at different scales the errors are statistical. The RGI values are computed from that one at $\mu a \simeq 1$ according to the eq. (23) and the first error is statistical, the second systematic as explained in text. 


\begin{tabular}{|c|c|c|c|c|c|c|}
\hline$\beta$ & $\mu^{2} a^{2}$ & $Z_{A}$ & $Z_{V}$ & $Z_{P}$ & $Z_{S}$ & $Z_{P} / Z_{S}$ \\
\hline \multirow{9}{*}{6.0} & 0.308 & $0.847(16)$ & $0.681(9)$ & $0.241(6)$ & $0.559(13)$ & $0.445(10)$ \\
\hline & 0.617 & $0.843(10)$ & $0.716(7)$ & $0.372(3)$ & $0.654(7)$ & $0.575(10)$ \\
\hline & 0.964 & $0.808(7)$ & $0.707(4)$ & $0.447(5)$ & $0.682(9)$ & $0.657(8)$ \\
\hline & 1.272 & $0.807(10)$ & $0.711(7)$ & $0.492(6)$ & $0.713(10)$ & $0.692(10)$ \\
\hline & 1.388 & $0.804(8)$ & $0.705(5)$ & $0.503(4)$ & $0.725(10)$ & $0.695(10)$ \\
\hline & 1.851 & $0.795(7)$ & $0.710(4)$ & $0.549(3)$ & $0.746(10)$ & $0.736(9)$ \\
\hline & 2.467 & $0.797(7)$ & $0.722(5)$ & $0.588(3)$ & $0.762(7)$ & $0.772(7)$ \\
\hline & 4.010 & $0.809(4)$ & $0.744(3)$ & $0.649(2)$ & $0.795(5)$ & $0.816(4)$ \\
\hline & RGI & $0.808(7)(7)$ & $0.707(4)(19)$ & $0.294(3)(39)$ & $0.448(6)(5)$ & $0.657(8)(80)$ \\
\hline \multirow{9}{*}{6.2} & 0.308 & $0.841(8)$ & $0.719(8)$ & $0.272(8)$ & $0.615(21)$ & $0.463(25)$ \\
\hline & 0.617 & $0.846(8)$ & $0.749(6)$ & $0.436(11)$ & $0.708(8)$ & $0.629(19)$ \\
\hline & 0.964 & $0.812(6)$ & $0.724(4)$ & $0.499(5)$ & $0.722(7)$ & $0.695(12)$ \\
\hline & 1.272 & $0.820(6)$ & $0.734(3)$ & $0.539(4)$ & $0.755(7)$ & $0.718(8)$ \\
\hline & 1.388 & $0.813(3)$ & $0.732(3)$ & $0.548(5)$ & $0.755(8)$ & $0.742(10)$ \\
\hline & 1.851 & $0.804(3)$ & $0.728(3)$ & $0.580(3)$ & $0.768(5)$ & $0.761(6)$ \\
\hline & 2.467 & $0.808(4)$ & $0.739(4)$ & $0.621(3)$ & $0.787(4)$ & $0.790(5)$ \\
\hline & 4.010 & $0.816(4)$ & $0.759(3)$ & $0.675(3)$ & $0.810(4)$ & $0.833(4)$ \\
\hline & RGI & $0.812(6)(8)$ & $0.724(4)(17)$ & $0.310(3)(31)$ & $0.448(5)(5)$ & $0.695(12)(69)$ \\
\hline \multirow{9}{*}{6.4} & 0.313 & $0.843(7)$ & $0.752(6)$ & $0.413(7)$ & $0.649(9)$ & $0.643(14)$ \\
\hline & 0.617 & $0.831(8)$ & $0.747(6)$ & $0.516(5)$ & $0.710(12)$ & $0.730(12)$ \\
\hline & 0.964 & $0.825(6)$ & $0.748(5)$ & $0.572(4)$ & $0.742(7)$ & $0.773(8)$ \\
\hline & 1.169 & $0.820(6)$ & $0.745(6)$ & $0.590(5)$ & $0.754(6)$ & $0.785(7)$ \\
\hline & 1.439 & $0.824(6)$ & $0.753(4)$ & $0.617(5)$ & $0.774(6)$ & $0.797(7)$ \\
\hline & 1.782 & $0.823(7)$ & $0.755(6)$ & $0.637(5)$ & $0.787(7)$ & $0.810(6)$ \\
\hline & 2.467 & $0.825(7)$ & $0.762(5)$ & $0.669(5)$ & $0.803(7)$ & $0.833(4)$ \\
\hline & 3.740 & $0.840(5)$ & $0.784(5)$ & $0.710(5)$ & $0.830(6)$ & $0.855(3)$ \\
\hline & RGI & $0.825(6)(10)$ & $0.748(5)(20)$ & $0.333(2)(21)$ & $0.431(4)(4)$ & $0.773(8)(41)$ \\
\hline
\end{tabular}

Table 3

Non-perturbative values of $Z_{\Gamma}^{\mathrm{RI}}\left(m_{q}=0\right)$ with the Wilson action, for all couplings at several renormalization scales $\mu^{2} a^{2}$. For the values at different scales the errors are statistical. The RGI values are computed from that one at $\mu a \simeq 1$ according to the eq. (23) and the first error is statistical, the second systematic as explained in text. 


\begin{tabular}{|c|c|c|c|c|c|c|c|}
\hline Action & $\beta$ & Method & $Z_{A}$ & $Z_{V}$ & $Z_{P}$ & $Z_{S}$ & $Z_{P} / Z_{S}$ \\
\hline & 6.0 & $\mathrm{SPT}$ & 0.98 & 0.90 & 0.74 & 0.91 & 0.82 \\
\hline & 6.0 & $\mathrm{BPT}$ & 0.97 & 0.83 & 0.56 & 0.84 & 0.67 \\
\hline & 6.0 & WI [31] & $1.10(2)$ & $0.80(2)$ & & & $0.61(2)$ \\
\hline & 6.0 & [This work] & $1.05(3)$ & $0.85(1)$ & $0.41(6)$ & $0.83(2)$ & $0.49(6)$ \\
\hline \multirow[t]{11}{*}{ SW } & 6.2 & SPT & 0.98 & 0.90 & 0.75 & 0.91 & 0.83 \\
\hline & 6.2 & $\mathrm{BPT}$ & 0.97 & 0.84 & 0.59 & 0.85 & 0.70 \\
\hline & 6.2 & WI [32] & $1.05(1)$ & $0.82(1)$ & & & $0.69(4)$ \\
\hline & 6.2 & [This work] & $1.02(2)$ & $0.85(1)$ & $0.47(5)$ & $0.85(2)$ & $0.56(5)$ \\
\hline & 6.4 & $\mathrm{SPT}$ & 0.98 & 0.91 & 0.76 & 0.91 & 0.83 \\
\hline & 6.4 & $\mathrm{BPT}$ & 0.97 & 0.85 & 0.62 & 0.86 & 0.72 \\
\hline & 6.4 & [This work] & $1.01(1)$ & $0.863(7)$ & $0.55(3)$ & $0.85(2)$ & $0.65(2)$ \\
\hline & 6.0 & $\mathrm{SPT}$ & 0.87 & 0.83 & 0.78 & 0.86 & 0.91 \\
\hline & 6.0 & $\mathrm{BPT}$ & 0.78 & 0.71 & 0.62 & 0.76 & 0.82 \\
\hline & 6.0 & WI [2] & $0.85(7)$ & $0.79(4)$ & & & \\
\hline & 6.0 & [This work] & $0.81(1)$ & $0.71(2)$ & $0.45(6)$ & $0.68(1)$ & $0.66(8)$ \\
\hline \multirow[t]{7}{*}{ Wilson } & 6.2 & $\mathrm{SPT}$ & 0.87 & 0.83 & 0.78 & 0.86 & 0.91 \\
\hline & 6.2 & $\mathrm{BPT}$ & 0.79 & 0.73 & 0.65 & 0.73 & 0.84 \\
\hline & 6.2 & [This work] & $0.81(1)$ & $0.72(2)$ & $0.50(5)$ & $0.72(1)$ & $0.69(7)$ \\
\hline & 6.4 & $\mathrm{SPT}$ & 0.87 & 0.84 & 0.79 & 0.86 & 0.91 \\
\hline & 6.4 & $\mathrm{BPT}$ & 0.80 & 0.74 & 0.67 & 0.79 & 0.85 \\
\hline & 6.4 & WI [31] & & $0.71(1)$ & & & \\
\hline & 6.4 & [This work] & $0.82(1)$ & $0.75(2)$ & $0.57(4)$ & $0.74(1)$ & $0.77(5)$ \\
\hline
\end{tabular}

Table 4

Perturbative values (Standard and Boosted PT) of $Z_{\Gamma}^{\mathrm{RI}}\left(m_{q}=0\right)$ and nonperturbative values from the WI with the SW and Wilson action. The perturbative $Z$ 's are evaluated at $\mu^{2} a^{2}=1$.

While $Z_{P}$ and $Z_{S}$ cannot be determined separately by imposing a Ward Identity, their ratio $Z_{P} / Z_{S}$ can as it is scale independent. For this reason it can be treated in a similar fashion to $Z_{A}$ and $Z_{V}$ and can be compared with the values obtained from the WI. In fig. 5 we show $Z_{P} / Z_{S}$ as function of $\mu$. As expected, the behaviour of the ratio is dominated by the behaviour of $Z_{P}$ and agreement with the WI determination seems to be obtained at rather high values of the renormalization scale, at which discretization effects should dominate. 

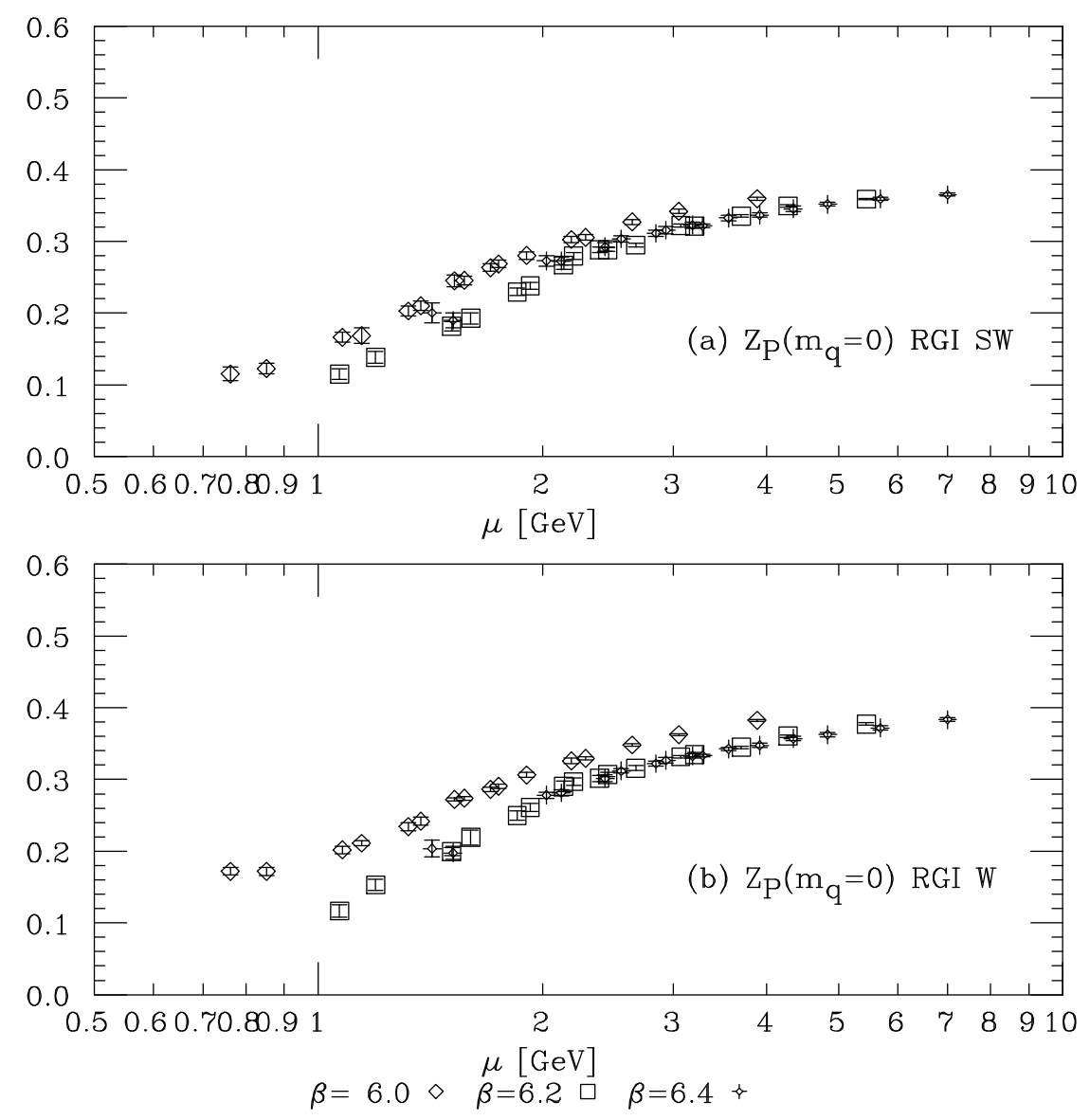

Fig. 3. RGI values for the renormalization of the pseudoscalar density for (a) SW and (b) Wilson action as a function of $\mu$ for all couplings. The lattice spacing is determined from $M_{K^{*}}$.

\section{Meson decay constants}

There are several interesting phenomenological quantities which can be extracted from the matrix elements of quark bilinears, such as leptonic decay constants and quark masses. The problem of a non-perturbative measurement of quark masses has been addressed in [11] to which we refer the reader for all details. A very interesting by-product of both quark masses and decay constants is the estimate of the chiral condensate, which is of great phenomenological relevance. The issue will be addressed in a forthcoming paper [12], together with a detailed theoretical analysis.

In the remainder of this section, we concentrate on the determination of the leptonic decay constants of mesons. The pseudoscalar and vector decay constants, $f_{P S}$ and $f_{V}$, are defined as 

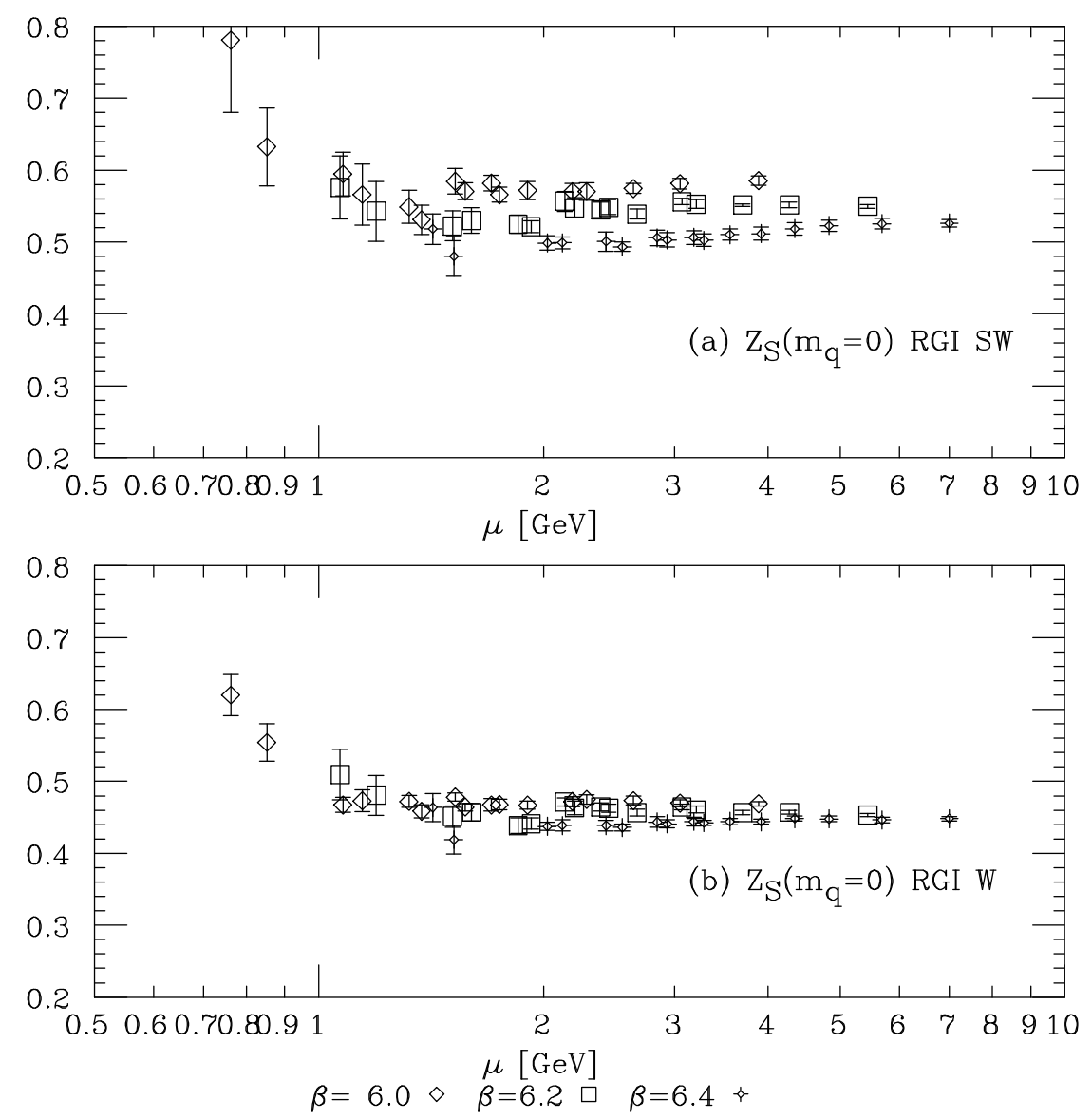

Fig. 4. RGI values for the renormalization of the scalar density for (a) SW and (b) Wilson action as a function of $\mu$ for all couplings. The lattice spacing is determined from $M_{K^{*}}$.

$$
\begin{aligned}
\left\langle 0\left|A_{0}\right| P S\right\rangle & =i \frac{f_{P S}}{Z_{A}} M_{P S}, \\
\left\langle 0\left|V_{i}\right| V, r\right\rangle & =\epsilon_{i}^{r} \frac{M_{V}^{2}}{f_{V} Z_{V}},
\end{aligned}
$$

where $\epsilon_{i}^{r}$ is the vector-meson polarization, $M_{P S}$ and $M_{V}$ are the pseudoscalar and vector masses, $A_{0}$ and $V_{i}$ the temporal and spatial components of the axial and vector currents respectively, and $Z_{V, A}$ the corresponding renormalization constants.

In tab. 5 we summarize the parameters used in the simulations. The bare lattice decay constants have been extracted from the appropriate correlation functions as described in ref. [34], to which we refer for details. In tab. 6 and 7 we present the results for the decay constants, both the bare unrenormalized values in lattice units and the renormalized ones in physical units, obtained using the renormalization constants at $\mu a \simeq 1$. In the estimate of the error on renormalized decay constants we have neglected the errors on the 

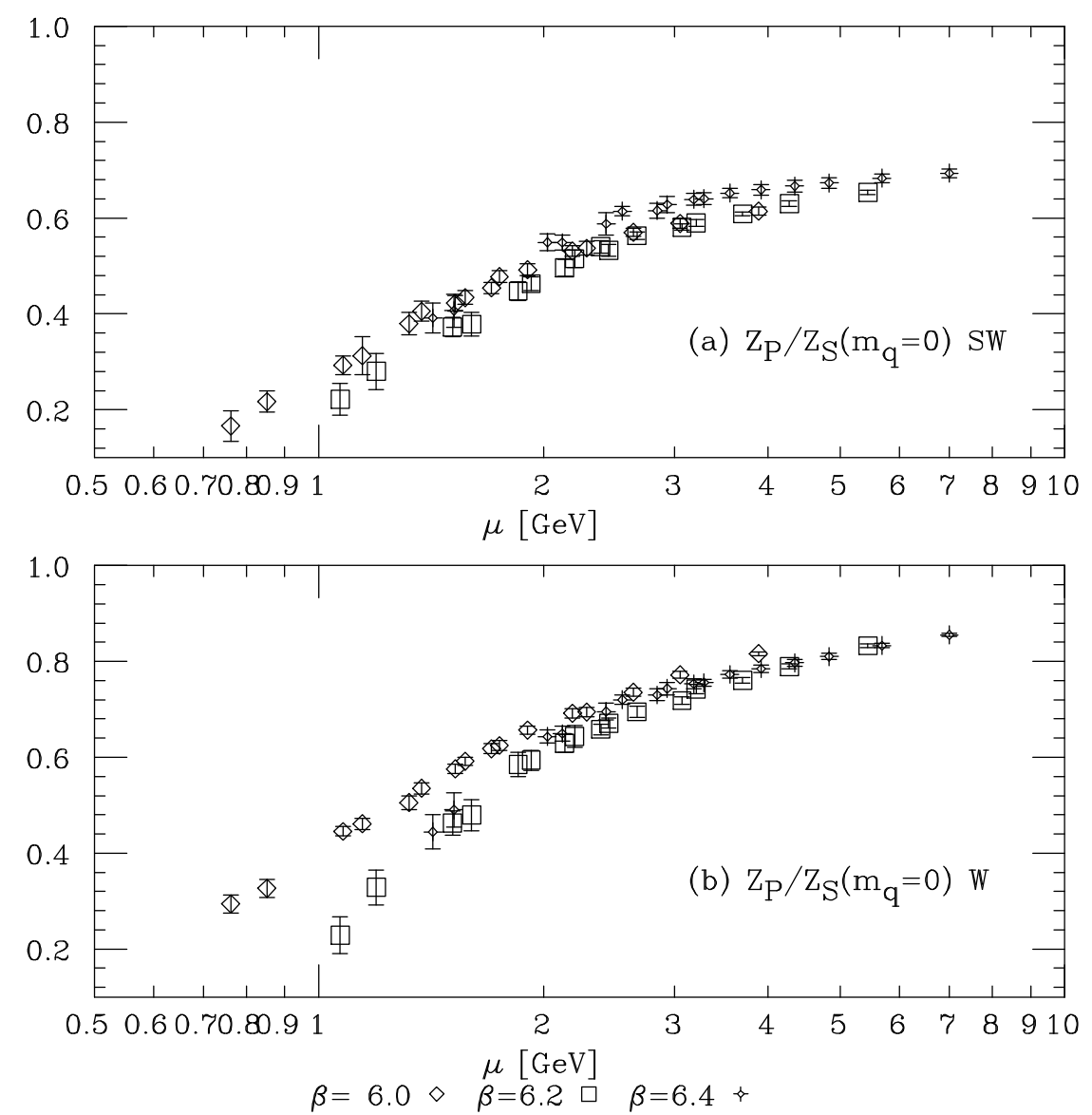

Fig. 5. Renormalization of the pseudoscalar to scalar density ratio for (a) SW and (b) Wilson action as a function of $\mu$ for all couplings. The lattice spacing is determined from $M_{K^{*}}$.

renormalization constants. The non-perturbatively renormalized values and the experimental values present roughly a $10-15 \%$ discrepancy, which is to be expected, considering that we have performed all calculations in the quenched approximation. It is important, though, to note that compared to the values obtained with perturbative renormalization in ref. [34], the values obtained with a non-perturbative renormalization are in general closer to the experimental results. We have not attempted an extrapolation to the continuum limit as the physical volume at the smallest coupling is too small to confidently extract hadronic matrix elements.

\section{Conclusions}

In this paper we have performed a systematic study of the renormalization of quark bilinears, in a non-perturbative fashion. We have also analyzed the discretization effects, by performing our calculations with two different actions, 


\begin{tabular}{|c|cccccc|}
\hline \hline$\beta$ & 6.0 & 6.0 & 6.2 & 6.2 & 6.4 & 6.4 \\
Action & SW & Wilson & SW & Wilson & SW & Wilson \\
\# Confs & 490 & 320 & 250 & 250 & 400 & 400 \\
Volume & $18^{3} \times 64$ & $18^{3} \times 64$ & $24^{3} \times 64$ & $24^{3} \times 64$ & $24^{3} \times 64$ & $24^{3} \times 64$ \\
\hline$\kappa$ & 0.1425 & 0.1530 & 0.14144 & 0.1510 & 0.1400 & 0.1488 \\
& 0.1432 & 0.1540 & 0.14184 & 0.1515 & 0.1403 & 0.1492 \\
& 0.1440 & 0.1550 & 0.14224 & 0.1520 & 0.1406 & 0.1496 \\
& & & 0.14264 & 0.1526 & 0.1409 & 0.1500 \\
\hline$t_{1}-t_{2}$ & $15-28$ & $15-28$ & $18-28$ & $18-28$ & $24-30$ & $24-30$ \\
\hline$a^{-1}\left(K^{*}\right)$ & $2.123(62)$ & $2.258(50)$ & $2.719(141)$ & $2.993(94)$ & $4.004(195)$ & $4.149(161)$ \\
\hline \hline
\end{tabular}

Table 5

Summary of the parameters used in the calculation of the matrix elements.

\begin{tabular}{|l|cccccc|}
\hline \hline$\beta$ & 6.0 & 6.0 & 6.2 & 6.2 & 6.4 & 6.4 \\
Action & SW & Wilson & SW & Wilson & SW & Wilson \\
\hline$\left(f_{K} a\right) / Z_{A}$ & $0.0735(18)$ & $0.0944(26)$ & $0.0540(23)$ & $0.0640(21)$ & $0.0406(14)$ & $0.0480(16)$ \\
$\left(f_{\pi} a\right) / Z_{A}$ & $0.0661(21)$ & $0.0878(31)$ & $0.0470(28)$ & $0.0568(24)$ & $0.0370(16)$ & $0.0438(19)$ \\
\hline $1 /\left(f_{\phi} Z_{V}\right)$ & $0.348(9)$ & $0.451(11)$ & $0.332(8)$ & $0.417(8)$ & $0.284(9)$ & $0.363(8)$ \\
$1 /\left(f_{K^{*}} Z_{V}\right)$ & $0.366(14)$ & $0.482(16)$ & $0.359(20)$ & $0.446(15)$ & $0.290(13)$ & $0.377(12)$ \\
$1 /\left(f_{\rho} Z_{V}\right)$ & $0.384(20)$ & $0.513(21)$ & $0.386(33)$ & $0.475(22)$ & $0.297(17)$ & $0.391(17)$ \\
\hline \hline
\end{tabular}

Table 6

Lattice bare decay constants for all couplings and both actions.

\begin{tabular}{|lc|cccccc|}
\hline \hline$\beta$ & $\operatorname{Exp}$ & 6.0 & 6.0 & 6.2 & 6.2 & 6.4 & 6.4 \\
Action & & SW & Wilson & SW & Wilson & SW & Wilson \\
\hline$f_{K}$ & 0.1598 & $0.163(12)$ & $0.172(6)$ & $0.150(8)$ & $0.155(5)$ & $0.164(9)$ & $0.164(8)$ \\
$f_{\pi}$ & 0.1307 & $0.147(12)$ & $0.160(7)$ & $0.131(10)$ & $0.138(6)$ & $0.150(10)$ & $0.150(10)$ \\
\hline $1 / f_{\phi}$ & 0.23 & $0.294(8)$ & $0.319(8)$ & $0.281(7)$ & $0.302(6)$ & $0.245(8)$ & $0.271(6)$ \\
$1 / f_{K^{*}}$ & & $0.309(12)$ & $0.341(11)$ & $0.304(17)$ & $0.323(11)$ & $0.251(11)$ & $0.282(9)$ \\
$1 / f_{\rho}$ & 0.28 & $0.324(17)$ & $0.363(15)$ & $0.327(28)$ & $0.344(16)$ & $0.256(14)$ & $0.293(12)$ \\
\hline \hline
\end{tabular}

Table 7

Non-perturbatively renormalized decay constants (in $\mathrm{GeV}$ ) for all couplings and both actions.

the standard Wilson action and the tree-level improved SW-Clover action, at three different values of the couplings. We have performed a RG analysis at 
the NNLO and defined RGI values for the scale dependent renormalization constants. We have also used this approach to estimate the systematic error induced by discretization. Finally, we have applied our results to the calculation of the pseudoscalar and vector decay constants and we find that the non-perturbatively renormalized values, albeit with still sizable statistical errors, show a trend towards the experimental values with respect to the ones obtained with perturbative renormalization. With our data an extrapolation to the continuum limit is not reliable as the physical volume at the smallest coupling is too small to confidently extract hadronic matrix elements.

\section{Acknowledgements}

We warmly thank V. Lubicz, G. Martinelli, M. Testa and A. Vladikas for enlightening discussions. MT acknowledges the support of PPARC through grant GR/L22744. 


\section{References}

[1] M. Bochicchio, L. Maiani, G. Martinelli, G.C. Rossi and M. Testa, Nucl. Phys. B 262 (1985) 331.

[2] L. Maiani and G. Martinelli, Phys. Lett. B 178 (1986) 265.

[3] G. Martinelli and Y.C. Zhang, Phys. Lett. B 123 (1983) 433.

B. Meyer and C. Smith, Phys. Lett. B 123 (1983) 62.

[4] A. Borrelli, C. Pittori, R. Frezzotti and E. Gabrielli, Nucl. Phys. B 409 (1993) 382.

[5] G. Parisi, in "High Energy Physics - 1980", Proceedings of the XXth International Conference, Madison, Wisconsin, eds. L. Durand and L. G. Pondrom (American Institute of Physics, New York, 1981).

[6] G.P. Lepage and P.B. Mackenzie, Phys. Rev. D48 (1993) 2250.

[7] G. Martinelli, C. Pittori, C.T. Sachrajda, M. Testa and A. Vladikas, Nucl. Phys. B 445 (1995) 81.

[8] K. Jansen et al., Phys. Lett. B 372 (1996) 275.

[9] M. Ciuchini, E. Franco, G. Martinelli, L. Reina, L. Silvestrini, Z. Phys. C 68 (1995) 239.

[10] G. Martinelli, S. Petrarca, C.T. Sachrajda and A. Vladikas, Phys. Lett. B 311 (1993) 241, E: B317 (1993) 660.

[11] C. R. Allton et al., Nucl. Phys. B431 (1994) 667.

V. Giménez, L. Giusti, F. Rapuano and M. Talevi, hep-lat/9801028

[12] L. Giusti, F. Rapuano, M. Talevi, A. Vladikas, in preparation.

[13] A. Donini, G. Martinelli, C.T. Sachrajda, M. Talevi and A. Vladikas, Phys. Lett. B360 (1995) 83.

[14] L. Conti, A. Donini, V. Giménez, G. Martinelli, M. Talevi and A. Vladikas, Phys. Lett. B421 (1998) 273.

[15] S. Aoki et al. (JLQCD Collaboration), Nucl. Phys. (Proc. Suppl.) B 60A (1998) 67.

[16] C. Dawson, G. Martinelli, G.C. Rossi, C.T. Sachrajda, S. Sharpe, M. Talevi and M. Testa, Nucl. Phys. B 514 (1998) 313.

[17] B. Sheikholeslami and R. Wohlert, Nucl. Phys. B 259 (1985) 572.

G. Heatlie, G. Martinelli, C. Pittori, G.C. Rossi and C.T. Sachrajda, Nucl. Phys. B 352 (1991) 266. 
[18] K. Symanzik, Nucl. Phys. B 226 (1983) 187 and 205.

M. Lüscher and P. Weisz, Commun. Math. Phys. 97 (1985) 59, E: Commun. Math. Phys. 98 (1985) 433.

[19] M. Lüscher, S. Sint, R. Sommer and P. Weisz, Nucl. Phys. B 478 (1996) 365.

M. Lüscher, S. Sint, R. Sommer, P. Weisz and U. Wolff, Nucl. Phys. B 491 (1997) 323.

M. Lüscher, S. Sint, R. Sommer and H. Wittig, Nucl. Phys. B 491 (1997) 344.

[20] S. Capitani, M. Guagnelli, M. Lüscher, S. Sint, R. Sommer, P. Weisz and H. Wittig, Nucl. Phys. B (Proc. Suppl.) 63 (1998) 153.

[21] G. Martinelli, G.C. Rossi, C.T. Sachrajda, S. Sharpe, M. Talevi and M. Testa. Phys. Lett. B 411 (1997) 141.

[22] S. Sharpe, Nucl. Phys. B (Proc. Suppl.) 53 (1997) 181.

[23] M. L. Paciello, S. Petrarca, B. Taglienti and A. Vladikas, Phys. Lett. B 341 (1994) 187.

[24] C. Parrinello, S. Petrarca and A. Vladikas, Phys. Lett. B 268 (1991) 236.

[25] L. Giusti, Nucl. Phys. B 498 (1997) 331.

L. Giusti, M.L. Paciello, S. Petrarca, B. Taglienti and M. Testa, hep-lat/9803021

[26] E. Franco and V. Lubicz, hep-ph/9803491.

[27] T. van Ritbergen, J.A.M. Vermaseren and S.A. Larin, Phys. Lett. B400 (1997) 379.

[28] J.A.M. Vermaseren, S.A. Larin and T. van Ritbergen, Phys. Lett. B405 (1997) 327.

[29] K.G. Chetyrkin, Phys. Lett. B404 (1997) 161.

[30] R. Tarrach, Nucl. Phys. B183 (1981) 384.

[31] M. Crisafulli, V. Lubicz and A. Vladikas, hep-lat/9707025, to appear in Eur. Phys. J. C.

[32] D.S. Henty et al. (UKQCD Collaboration), Phys. Rev. D51 (1995) 5323.

[33] M. Göckeler et al., Nucl. Phys. B (Proc. Suppl.) 63 (1998) 868.

H. Oelrich, private communication.

[34] C. R. Allton, V. Giménez, L. Giusti and F. Rapuano, Nucl. Phys. B489 (1997) 427. 